\title{
Ablation Front Rayleigh- Taylor Growth Experiments in Spherically Convergent Geometry
}

S.G. Glendinning, C. Cherfils, J. Colvin, L. Divol, D. Galmiche, S. Haan, M. Marinak, B.A. Remington, A.L. Richard, and R. Wallace

This article was submitted to the $41^{\text {st }}$ Annual Meeting of the Division of Plasma Physics, Seattle, WA, November 15-19, 1999

U.S. Department of Energy

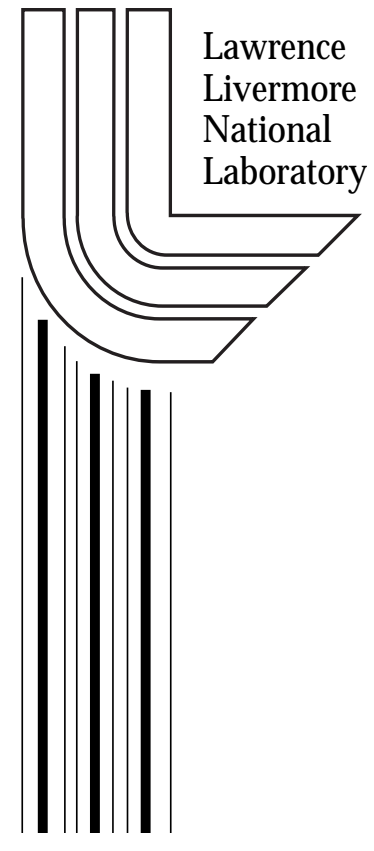

November 3, 1999 


\section{DISCLAIMER}

This document was prepared as an account of work sponsored by an agency of the United States Government. Neither the United States Government nor the University of California nor any of their employees, makes any warranty, express or implied, or assumes any legal liability or responsibility for the accuracy, completeness, or usefulness of any information, apparatus, product, or process disclosed, or represents that its use would not infringe privately owned rights. Reference herein to any specific commercial product, process, or service by trade name, trademark, manufacturer, or otherwise, does not necessarily constitute or imply its endorsement, recommendation, or favoring by the United States Government or the University of California. The views and opinions of authors expressed herein do not necessarily state or reflect those of the United States Government or the University of California, and shall not be used for advertising or product endorsement purposes.

This is a preprint of a paper intended for publication in a journal or proceedings. Since changes may be made before publication, this preprint is made available with the understanding that it will not be cited or reproduced without the permission of the author.

This report has been reproduced directly from the best available copy.

Available electronically at http://www.doc.gov/bridge

Available for a processing fee to U.S. Department of Energy

And its contractors in paper from

U.S. Department of Energy

Office of Scientific and Technical Information

P.O. Box 62

Oak Ridge, TN 37831-0062

Telephone: (865) 576-8401

Facsimile: (865) 576-5728

E-mail: reports@adonis.osti.gov

Available for the sale to the public from

U.S. Department of Commerce

National Technical Information Service

5285 Port Royal Road

Springfield, VA 22161

Telephone: (800) 553-6847

Facsimile: (703) 605-6900

E-mail: orders@ntis.fedworld.gov

Online ordering: http://www.ntis.gov/ordering.htm

OR

Lawrence Livermore National Laboratory

Technical Information Department's Digital Library

http://www.llnl.gov/tid/Library.html 


\section{Ablation Front Rayleigh-Taylor Growth Experiments in Spherically Convergent Geometry}

S. G. Glendinning ${ }^{1}$, C. Cherfils ${ }^{2}$, J. Colvin ${ }^{1}$, N. Dague ${ }^{2}$, L. Divol ${ }^{2}$, D. Galmiche ${ }^{2}$, S. Haan ${ }^{1}$, D H Kalantar ${ }^{1}$, O. L. Landen ${ }^{1}$, M. M. Marinak ${ }^{1}$, B. A. Remington ${ }^{1}$, A. L. Richard ${ }^{2}$, R. Wallace ${ }^{1}$ ${ }^{1}$ Lawrence Livermore National Laboratory, P.O. Box 808, Livermore, CA 94551, US

${ }^{2}$ Commissariat a l'Energie Atomique - Direction des Applications Militaire Ile de France, BP 12, 91680 Bruyeres le Chatel, France 
Abstract: Experiments were performed on the Nova laser [E.M. Campbell et al., Review of Scientific Instruments 57, 2101 (1986)], using indirectly driven capsules mounted in cylindrical gold hohlraums, to measure the Rayleigh-Taylor growth at the ablation front by time-resolved radiography. Modulations were preformed on the surface of Ge-doped plastic capsules. With initial modulation amplitude of 2-2.5 $\mu \mathrm{m}$, growth factors of about 6 in optical depth were seen, in agreement with simulations using the radiation hydrocode FCI2[G. Schurtz, La fusion thermonucleaire inertielle par laser. R. Dautray et al., Eds., (Eyrolles, 1994), vol. 2.]. With initial modulation amplitude of $0.5 \mu \mathrm{m}$ and a longer drive, growth factors of about 100-150 in optical depth were seen. Comparable planar experiments showed growth factors of about 40 in optical depth. Analytical models predict the observed growth factors. 


\section{Introduction}

The Rayleigh-Taylor (RT) instability at the ablation front in an inertial confinement fusion capsule has been the subject of considerable investigation. Much of this research has been concentrated on planar experiments, in which RT growth is inferred from radiography. The evolution is somewhat different in a converging geometry; the spatial wavelength decreases (affecting the onset of nonlinear saturation), and the shell thickens and compresses rather than decompressing as in a planar geometry. In a cylindrically convergent geometry, the latter effect is proportional to the radius, while in spherically convergent geometry, the latter effect is proportional to the radius squared ${ }^{1-3}$.

Past experiments were performed in cylindrical geometry 4,5 (using both direct and indirect drive). These experiments concentrated on imaging a buried tracer layer and examining the feed through of modulations during the stagnation of the imploding layer, rather than the growth at the ablation front as was the goal of the experiments reported here. Experiments have been performed in spherical geometry using direct drive 6,7 , using the same technique of transmission radiography which we have used.

Linear, classical theory may be used to understand the difference in growth between spherical and planar geometry. Using the terminology of Ref. 1, the expression for modulation growth at the outer surface of a compressible spherical shell is given by

$$
\ddot{\mathrm{c}}-\dot{\mathrm{c}}\left(\frac{\dot{\mathrm{R}}}{\mathrm{R}}+\frac{\dot{\rho}}{\rho}\right)+\ell c \frac{\ddot{R}}{\mathrm{R}}=0
$$

where $\boldsymbol{c}$ is the normalized amplitude (the spatial amplitude, $\eta$, divided by $\rho \mathrm{R}^{2}$ ), $\ell$ is the Legendre mode number, and $\mathrm{R}$ is the unperturbed interface position. We refer to this as Bell-Plesset theory. The equation without the term proportional to $\dot{c}$ is similar to the classical Rayleigh-Taylor growth in planar geometry, where $\ell / \mathrm{R}$ would be replaced by the wave number k. Early in the 
implosion, as convergence increases and $\mathrm{R}$ decreases, the difference between planar and convergent will appear as a higher linear growth rate for decreasing spatial wavelengths, followed by an earlier onset of nonlinear saturation in convergent than in planar geometry. The term proportional to $\dot{C}$ is unique to spherically convergent geometry and leads to increased growth. A more complete linear theory of the stability of ablation fronts in convergent geometry is found in Goncharov 8 .

This paper describes four different experiments comprising an examination of the effects of convergence in radiation-driven targets: two are convergent and two are similar planar geometry experiments for comparison. Three different experimental configurations were used. In both the planar experiments, a corrugated foil was placed at the wall of a cylindrical gold hohlraum, with corrugations facing the inside of the hohlraum. In one of the convergent experiments, the corrugated sphere was also mounted at the wall of the hohlraum (with the corrugations facing the drive), and thus driven as a hemispherical implosion. In the other implosion configuration the corrugated sphere was mounted at the center of the hohlraum for improved convergence. We report the results of the experiments here, and compare them with analytical expressions and with two-dimensional radiation hydrodynamic simulations performed with the Lagrangian codes FCI2 9,10 and LASNEX ${ }^{11}$.

\section{Experimental configurations}

The experimental configurations are shown in Figure 1. Eight beams of Nova were used to illuminate cylindrical gold hohlraums, with laser pulses of either 2.2 or $4.5 \mathrm{~ns}$ duration. The hohlraum, sample, and laser parameters for the different experiments are shown in Table I. Two diagnostic holes were cut in the hohlraums to allow face-on radiography of the capsule or foil. For brevity, we refer to the four configurations as P-26, C-26, P-35, and C-35 for the planar and convergent 2.2 ns (Nova "PS26") drive and planar and convergent 4.5 ns (Nova "PS35") drive throughout this paper. The laser pulses and resulting x-ray drives for the different cases are shown in Figure 2. The radiography diagnosis is provided by two Nova beams, which illuminated a separate target, either of rhodium, scandium, or titanium, providing X-rays between 
3 and $5 \mathrm{keV}$ depending on the target. For the P-26 and C-26 experiments, the backlighter material was rhodium., for the P-35 experiment it was scandium, and for the C-35 experiment it was titanium. The resulting (time-integrated) spectra were measured with a crystal spectrometer 12 and are shown in Figure 3. The primary diagnostic was one of two x-ray pinhole cameras coupled to a gated microchannel plate detector. The detector used for the C-26 and P-26 experiments had an effective x-ray gate time of $40 \mathrm{ps}^{13}$ (required because of the very fast implosion velocity in this experiment), that used for the C-35 experiments had an effective x-ray gate time of $100 \mathrm{ps},{ }^{14}$ and that used for the P-35 experiments 200 ps. ${ }^{14}$ The pinhole cameras were the same for all experiments, an array of $10 \mu \mathrm{m}$ diameter pinholes with a system magnification of 8 . The measured system modulation transfer functions (different for the two MCP detectors) are shown in Figure 4.

The foils and capsules all had modulations fabricated on one surface. The P-35 foils were simply pressed into machined molds with sinusoidal perturbations with wavelengths of 50 or $100 \mu \mathrm{m}$ and amplitudes of 0.5 and $1.0 \mu \mathrm{m}$ for the two wavelengths. The capsule corrugations for the $\mathrm{C}$ 26 and C-35 series were made by laser ablating the capsules with an excimer laser; the same excimer laser was used to make a kapton mold for the P-26 foils. The C-26 capsules and the P-26 foils had a square region $280 \mathrm{X} 280 \mu \mathrm{m}$ with four $70 \mu \mathrm{m}$ wavelength sinusoidal perturbations with an initial amplitude of $2 \mu \mathrm{m}$. The C-35 capsules had a square region $120^{\circ} \times 120^{\circ}$ with a section of a Legendre mode number (1) either 24 or 32, with a peak to valley modulation of $1 \mu \mathrm{m}$ at the center. Profilometer traces of the capsule modulations are shown in Figure 5.

\section{Experimental results}

Typical images from the experiments are shown in Figure 6. The images in the top are the P-26 and $\mathrm{C}-26$ at various times, and those in the bottom row the P-35 and C-35. The images show the evolution into the weakly nonlinear regime, as the dark (more opaque) spikes become narrower than the light (less opaque) bubbles. The convergent experiment images also show the decrease in wavelength due to convergence. 
The images are analyzed by first converting the film density to exposure, then taking profiles in natural logarithm of exposure perpendicular to the corrugations. (The negative of the natural logarithm of exposure is equivalent to optical depth if the unattenuated exposure is known; if not, the modulation in $-\ln$ (exposure) is still equivalent to the modulation in optical depth.) Typical profiles are plotted in Figure 7a-d for the four experiments. The images for the P-26, C-26, and P-35 experiments, with initially sinusoidal modulations, were decomposed into Fourier amplitudes. The images for the C-35 series, with nominally Legendre modes imposed on the surface, were analyzed for the total modulation (peak minus valley) at the center of the modulation pattern. The resulting measured modulations as a function of time are shown in Figures 8a-e.

The C-35 experiment had certain special requirements. Because it used a larger hohlraum and longer laser pulse than had been previously used for implosions, the time-dependent symmetry of the imploding capsule had not been determined. Asymmetric implosions would result in, at minimum, difficult to interpret images and distortion modes have been shown ${ }^{5}$ to couple to imposed spatial modulations in the nonlinear regime, further complicating the interpretation of the experiment. Therefore a pointing scan was used to determine the pointing of best symmetry. The symmetry was determined by the distortion of the ablation front (point of maximum increase in transmission) in the capsule images. The best pointing was predicted from an analytical model of Lind1 15, 16 and agreed with the measurement. Most of the distortion controllable by pointing is a Legendre mode 2; some distortion at mode 4 was measured at a low level (about 8-10\% of the radius at the latest times).

Another special feature of the C-35 experiments was the clear visibility of the imploding shell, as shown in Figure 9a. This allowed the determination of the density and thickness of the shell by Abel inversion 17 of the images. Because of the modulations on the capsules, which extend to the shell at $\theta=90^{\circ}$ and $270^{\circ}$ (where $\theta=0^{\circ}$ is the hohlraum axis), radial profiles were taken of $20^{\circ}$ sectors around $\theta=0^{\circ}$ and $180^{\circ}$ and the profiles at the two angles were averaged to reduce noise. The result of this process for one image is shown in Figure 9b. The assumption of spherical symmetry required for an Abel inversion is broken by the residual distortion present in the 
implosion even at best pointing. Numerical investigations of the effect of the distortion for the typical values for our experiment showed that the inferred shell thickness was increased at $0^{\circ}$ by about $15 \%$, and the inferred density reduced by about the same amount, over a shell with no $\mathrm{P}_{4}$ mode. By post-processing a capsule simulated using LASNEX in 1D to produce a predicted image, including diagnostic resolution, and then inverting this image in the same manner as the data, we produced a simulated density and shell thickness. The simulated and inferred density and shell thickness are shown in Figures $10 \mathrm{a}$ and b. Both the simulated and measured densities increase about a factor of 2, while the shell thickness increases about a factor of 3 .

\section{Numerical simulations}

Simulations were done with two radiation hydrodynamics codes, FCI2 and LASNEX.

Simulating the experimental x-ray radiographs was a multistep process, particularly for the C-26 configuration. In that case, we first estimated a mean radiation drive temperature on the wallmounted capsule using a 3D view factor code. This code included radiative transport but no hydrodynamics and allowed us to estimate the effect on the X-ray drive of non-axisymmetric laser beams. On top of the capsule, in the angular range $56^{\circ}$ to $90^{\circ}$ relative to the wall of the hohlraum, peak drive temperatures range between $195 \mathrm{eV}$ and $201 \mathrm{eV}$, and are quite similar to the peak temperatures obtained with the view factor code for the P-26 experiment. The average drive temperature obtained this way is in good agreement with the radiation temperature shown in Figure 2a.

We then used the 2D radiation hydrodynamics code FCI2 to simulate the hydrodynamic evolution of the capsules and foils. FCI2 is a 2D Lagrangian code with rezoning, including non local-thermodynamic-equilibrium (NLTE) atomic physics, heat conduction with flux limiting, and different radiation packages as described in Refs. 9, 10, 18

The planar experiment P-35 was simulated with the 2D radiation hydrodynamics code LASNEX. The drive for that experiment was obtained from a LASNEX simulation of a two-dimensional gold hohlraum, and the simulation of the perturbation evolution was similar to that described by Weber et al. 19 
An issue of particular concern was the effect of the gold hohlraum wall on the observed perturbation evolution in the C-26 experiment. We did an FCI2 simulation of the capsule surrounded by a gold washer. This simulation showed no disturbance of the observed region before $2.5 \mathrm{~ns}$ (after the experiment was over).

\section{Discussion}

The difference between planar and convergent geometry in the P-26 and C-26 series was evident during the observation period as the effect of shrinking wavelength. The onset of nonlinear saturation begins earlier in the C-26 experiment than in the P-26 experiment. This appears as the higher ratio of second harmonic to fundamental $(0.4$ in the convergent case at $t=1.7 \mathrm{~ns}$ compared with 0.1 in the planar case at the same time). This was a rather stable experiment, with growth factors of only about 6 at the ablation front, due to the high temperature. The rapid turnover in the observed modulation in the C-26 experiment after about $2 \mathrm{~ns}$ is due to a combination of experimental effects; first, as the wavelength shrinks, the modulation transfer function drops and the measured modulation drops; second, and as the implosion velocity increases, motion blurring also reduces the measured modulation; and finally, as the shell thickens the higher energy part of the rhodium spectrum reduces the measured contrast. The simulated modulations at the ablation front continue to grow throughout the measurement. The turnover observed in the modulations in the $\mathrm{P}-26$ experiment is due to a combination of the reduction in growth rate due to nonlinear saturation and the foil decompression. This limitation in the observation during a crucial part of the implosion, and the low growth factors, prompted the change to the larger capsule and longer time scales of the P-35 and C-35 experiments. In those experiments the difference between planar and convergent is more dramatic.

The low-order distortions in the capsule shells measured in the C-35 experiment during the implosion may have an effect on the growth of pre-imposed perturbations in the nonlinear

regime due to mode coupling 4,20 . To second order, the coupling between modes may be estimated from

$$
\eta_{\mathrm{k}_{\mathrm{i}} \pm \mathrm{k}_{\mathrm{j}}} \approx \mp \frac{1}{2}\left(\mathrm{k}_{\mathrm{i}} \pm \mathrm{k}_{\mathrm{j}}\right) \eta_{\mathrm{k}_{\mathrm{i}}}^{\mathrm{L}} \eta_{\mathrm{k}_{\mathrm{j}}}^{\mathrm{L}}
$$


The modes seeded by the drive asymmetry at $5.5 \mathrm{~ns}$ ( $250 \mu \mathrm{m}$ displacement) in the C-35 experiment are Legendre modes 2 and 4, with measured spatial amplitudes about -7 and $+15 \mu \mathrm{m}$, respectively. Near $\theta=90^{\circ}$, where the preimposed mode is measured, these may be approximated by cosines with amplitudes $-5 \mu \mathrm{m}$ and $+6 \mu \mathrm{m}$. The mode 32 at $5.5 \mathrm{~ns}$ had an amplitude of about $+50 \mu \mathrm{m}$. The coupled modes predicted from Equation 2 are 6, 28, 30, 34, and 36, with amplitudes $-0.6 \mu \mathrm{m},+28 \mu \mathrm{m},-25 \mu \mathrm{m},+28$, and $-36 \mu \mathrm{m}$. Because of the opposite phases of the coupled modes, the change in the modulation measured near $\theta=90^{\circ}$ (see Figure $7 \mathrm{~d}$ ) is about $6 \%$ (well within the uncertainty in the measurement). However, mode coupling will certainly affect the growth of the imposed modulation at angles some distance from $90^{\circ}$.

Spatial amplitudes for the C-35 and P-35 experiments may be calculated from the observed optical depth amplitudes by dividing out the simulated density (determined from 1D simulations) and the opacity to the Ti and Sc backlighters. The amplitude growth of the two experiments as a function of displacement are compared in Figure 11 (normalized to the initial amplitude because the $\lambda=100 \mu \mathrm{m}$ experiment had an initial amplitude of $1 \mu \mathrm{m})$. The mode 24 and $100 \mu \mathrm{m}$ experiments are shown in Figure 11a and the mode 32 and $50 \mu$ m experiments are shown in Figure 11b. The C-35 experiment shows about a factor of 2-3 more growth than the P-35 experiment. The final amplitude is about $40 \%$ of the wavelength for the P-35 $(50 \mu \mathrm{m})$ experiment and about $120 \%$ of the wavelength for the C-35 (mode 32) experiment.

Rayleigh-Taylor growth at the ablation front has been theoretically investigated by Takabe ${ }^{21}$ and Betti ${ }^{22}$. Betti showed that, for indirect drive, the growth in the linear regime may be parameterized by

$$
\gamma=\sqrt{\frac{\mathrm{kg}}{1+\mathrm{kL}}}-\beta \mathrm{kV}_{\mathrm{a}}
$$

where $\gamma$ is the linear regime exponential growth rate, $\mathrm{k}$ is the wave number, $\mathrm{g}$ is the acceleration, $\mathrm{L}$ is the density gradient scale length, $\beta$ is a parameter between 1 and 3 , and $\mathrm{V}_{\mathrm{a}}$ is the ablation velocity. We used $\beta=1.7$ for these experiments. For the P-35 experiment, the values may be 
extracted from the 1D LASNEX simulations; $\mathrm{L}$ is $4 \mu \mathrm{m}$ and $\mathrm{V}_{\mathrm{a}}$ is $1.75 \mu \mathrm{m} / \mathrm{ns}$ during the acceleration phase. The calculated acceleration varies during this time, so the growth is calculated by integrating $\ddot{\eta}-\gamma^{2} \eta=0$, where $\eta$ is the amplitude. This is compared with the P-35 amplitudes for 50 and $100 \mu \mathrm{m}$ in Figure 11 and agrees with the data. It was assumed that no growth occurs at the ablation front during the shock transit phase 23 .

An estimate may be made of the convergent growth from Equation 1 by substituting $\gamma^{2}$ from Equation 3 for the classical $\ell \ddot{R} / R$ in Equation 1 to account for ablative stabilization. The values for the parameters $\mathrm{L}$ and $\mathrm{V}_{\mathrm{a}}$, extracted from 1D simulations, are the same as for the P-35 experiment. The acceleration $\mathrm{g}$ is different and the calculated time dependence is used. Because of the large growth, the amplitudes are not small and enter the nonlinear regime. Making the assumption that after the amplitude reaches a value of 0.4 of the wavelength it grows with a constant velocity given by the velocity at that point, we calculate the growth curves labeled "B$\mathrm{P}$ " in Figures 11a and b. These curves also agree with the data.

The difference in growth between the P-35 and C-35 experiments is about a factor of 2 after 250 $\mu \mathrm{m}$ displacement; however, because of nonlinear saturation and differing acceleration, the difference cannot be completely attributed to convergence. Using Equations 1 and 3 for acceleration profiles from the C-35 experiment and assuming linear growth, the resultant converging shell was predicted to grow a factor of 4 more than a planar foil with the same initial wavelength (50 $\mu \mathrm{m}$, mode 32). During convergence, the mode 32 wavelength decreased from 78 $\mu \mathrm{m}$ to $31 \mu \mathrm{m}$. Equation 3 predicts an increase in growth rate for decreased wavelength. A planar experiment, calculated as above, with a decreasing wavelength would grow a factor of 1.2 more than one with a constant wavelength, so the convergent effects may be separated into about a factor of 1.2 due to decreasing wavelength and a factor of 3.4 due to convergent growth. The predicted growth is ablatively stabilized; a classical interface would grow about a factor of 4 more in either planar or convergent geometry than a stabilized one.

The P-26 and C-26 experiments used a higher temperature $\mathrm{x}$-ray drive, resulting in values for $\mathrm{L}$ and $\mathrm{V}_{\mathrm{a}}$ of about $15 \mu \mathrm{m}$ and $2.5 \mu \mathrm{m} / \mathrm{ns}$. Thus, the predicted growth in amplitude is $\sim 6-10$. Because 
of this stability, the difference predicted by Equation 3 between the P-26 and C-26 experiments was only about a factor of 2; about 1.7 of this was due to decreasing wavelength, and about a factor of 1.2 due to convergent growth. Our 2D simulations of these experiments in fact predicted about a factor of 1.9 due to decreasing wavelength and about 1.1-1.2 due to convergent growth (not an observable effect) ${ }^{24}$.

\section{Conclusion}

In summary, we have done a series of experiments designed to compare and contrast the behavior of Rayleigh-Taylor unstable foils and shells in planar and spherically convergent geometries. One set of experiments, using a laser drive of $2.2 \mathrm{~ns}$ duration, showed an effect consistent with the shrinking wavelength of the ablation front perturbation in convergent geometry when compared with similar planar experiments. A second set, designed to extend the duration of the measurement in the convergent geometry, used a $4.5 \mathrm{~ns}$ laser drive. In these experiments, significant differences in the amount of hydrodynamic growth in the ablation front perturbations were seen. The total amount of growth in the planar experiments was about a factor of 40. Similar convergent geometry experiments showed growth factors of about 150 , for a convergence of 2.5. Simple analytical theories predict that in the absence of nonlinear saturation, a convergent shell with convergence of 2.5 would be expected to grow about a factor of 4 more than a planar foil with identical acceleration.

This work was performed under the auspices of the U.S. Department of Energy by University of California Lawrence Livermore National Laboratory under contract No. W-7405-Eng-48. 
Figure captions.

Figure 1. Experimental configurations: a) backlighting configuration, showing planar foil on side of hohlraum, backlighter target, laser beams, and diagnostic; b) capsule at wall of hohlraum.

Figure 2. Laser pulses (left hand axes) and measured x-ray drive (right hand axes) for a) P-26 and C-26 experiment, b) P-35 experiment, and c) C-35 experiment.

Figure 3. Backlighter spectra, a) rhodium (used for P-26 and C-26), b) scandium (used for P-35), and titanium (used for C-35).

Figure 4. Modulation transfer function for the framing cameras used.

Figure 5. Modulations initially imposed on the capsules: a) $70 \mu \mathrm{m}$ sinusoidal pattern (C-26), b) Legendre mode 24 (C-35), and c) Legendre mode 32 (C-35).

Figure 6. Typical images from a) P-26, at $\mathrm{t}=1.5$ and $2.2 \mathrm{~ns}, \mathrm{~b}) \mathrm{C}-26$, at $\mathrm{t}=1.5$ and $2.2 \mathrm{~ns}, \mathrm{c}) \mathrm{P}-35$, at $\mathrm{t}=5$ and $6 \mathrm{~ns}$, and $\mathrm{d}) \mathrm{C}-35$, at $\mathrm{t}=5$ and $6 \mathrm{~ns}$.

Figure 7. Profiles from the images in Figure $6 \mathrm{a}, \mathrm{b}, \mathrm{c}$, and d.

Figure 8. Modulation in optical depth as a function of time. a) P-26 Fourier amplitudes of the 70 $\mu$ mavelength. b) C-26 Fourier amplitudes of the fundamental wavelength (70 $\mu$ m initially). c) P-35 Fourier amplitudes of the $50 \mu \mathrm{m}$ wavelength, and d) $70 \mu \mathrm{m}$ wavelength. e) C-35, Peak to valley modulation of the mode 24 initial modulation, and f) peak to valley for the mode 32 modulation experiment. The different symbols are the results from different shots and the lines are simulated results from FCI2 (solid) and LASNEX (dashed).

Figure 9. Abel inversion of shell from C-35 experiment. a) original image, b) $20^{\circ}$ sector averaged radial profile and inferred density as a function of radius. 
Figure 10. Results of Abel inversions of C-35 experiment, a) density and b) shell thickness, both as a function of shell displacement. The symbols are data and the lines are simulations from LASNEX.

Figure 11. Comparison of convergent and planar results for a) the mode 24 C-35 and the $100 \mu \mathrm{m}$ P-35 experiments and b) the mode $32 \mathrm{C}-35$ and $50 \mu \mathrm{m}$ P-35 experiments. The curves are predictions from analytical theory. The quantities plotted are growth factors (inferred spatial modulation divided by initial spatial modulation).

Table I. Parameters for the experiments described. P-26 and P-35 are planar geometry, 2.2 ns drive and $4.5 \mathrm{~ns}$ drive respectively. C-26 and C-35 are comparable spherical geometry, $2.2 \mathrm{~ns}$ drive and 4.5 ns drive.

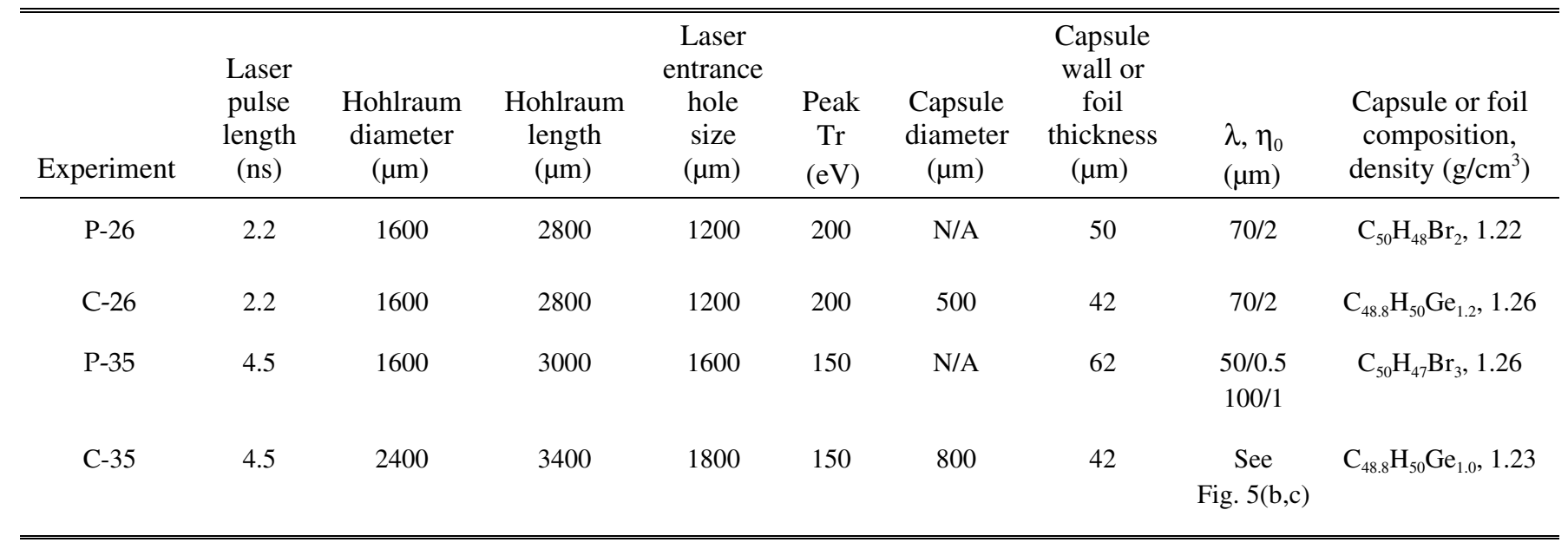


1. G.I. Bell, Los Alamos National Laboratory, Taylor instability on cylinders and spheres in the small amplitude approximation, LA-1321 (1951),.

2. G. Birkhoff, Quart. Appl. Math. 12, 306 (1952).

3. M.S.a.M. Plesset, T. P., Quart. Appl. Math. 13, 419 (1955).

4. W.W. Hsing, C.W. Barnes, J.B. Beck, N.M. Hoffman, D. Galmiche, A. Richard, J. Edwards, P. Graham, S. Rothman and B. Thomas, Physics of Plasmas 4, 1832 (1997).

5. D.L. Tubbs, C.W. Barnes, J.B. Beck, N.M. Hoffman, J.A. Oertel, R.G. Watt, T. Boehly, D. Bradley, P. Jaanimagi and J. Knauer, Physics of Plasmas 6, 2095 (1999).

6. J.S. Wark, J.D. Kilkenny, A.J. Cole, M.H. Key and P.T. Rumsby, Applied Physics Letters 48, 969 (1986).

7. H. Nishimura, H. Takabe, K. Mima, F. Hattori, H. Hasegawa, H. Azechi, M. Nakai, K. Kondo, T. Norimatsu, Y. Izawa, C. Yamanaka and S. Nakai, Physics of Fluids 31, 2875 (1988).

8. V.N. Goncharov, PhD, University of Rochester (1998).

9. G. Schurtz, La fusion thermonucleaire inertielle par laser. R. e. a. Dautray, Eds., (Eyrolles, 1994), vol. 2.

10. M. Busquet, Physics of Fluids B 5, 4191 (1993).

11. G.B. Zimmerman and W.L. Kruer, Comments on Plasma Physics and Controlled Fusion 2, 51 (1975).

12. L.N. Koppel, X-ray instrumentation in the LLL laser-fusion program, J. M. Weinberg and T. Hirschfelds, Eds., Unconventional spectroscopy (Soc. Photo-Optical Instrumentation Engrs, San Diego, CA, USA, 1976), pp. 75.

13. D.K. Bradley, P.M. Bell, O.L. Landen, J.D. Kilkenny and J. Oertel, Review of Scientific Instruments 66, 716 (1995).

14. K.S. Budil, T.S. Perry, P.M. Bell, J.D. Hares, P.L. Miller, T.A. Peyser, R. Wallace, H. Louis and D.E. Smith, Review of Scientific Instruments 67, 485 (1996).

15. J.D. Lindl, Inertial confinement fusion : the quest for ignition and energy gain using indirect drive (AIP Press ; Springer, New York, NY, USA, 1998).

16. O.L. Landen, P.A. Amendt, L.J. Suter, R.E. Turner, S.G. Glendinning, S.W. Haan, S.M. Pollaine, B.A. Hammel, M. Tabak, M.D. Rosen and J.D. Lindl, Physics of Plasmas 6, 2137 (1999). 
17. D.H. Kalantar, S.W. Haan, B.A. Hammel, C.J. Keane, O.L. Landen and D.H. Munro, Review of Scientific Instruments 68, 814 (1997).

18. E. Buresi, J. Coutant, R. Dautray, M. Decroisette, B. Duborgel, P. Guillaneux, J. Launspach, P. Nelson, C. Patou, J.M. Reisse and J.P. Watteau, Laser and Particle Beams 4, 531 (1985).

19. S.V. Weber, B.A. Remington, S.W. Haan, B.G. Wilson and J.K. Nash, Physics of Plasmas 1, 3652 (1994).

20. B.A. Remington, S.V. Weber, M.M. Marinak, S.W. Haan, J.D. Kilkenny, R.J. Wallace and G. Dimonte, Physics of Plasmas 2, 241 (1995).

21. H. Takabe, K. Mima, L. Montierth and R.L. Morse, Physics of Fluids 28, 3676 (1985).

22. R. Betti, V.N. Goncharov, R.L. McCrory and C.P. Verdon, Physics of Plasmas 5, 1446 (1998).

23. T. Endo, K. Shigemori, H. Azechi, A. Nishiguchi, K. Mima, M. Sato, M. Nakai, S. Nakaji, N. Miyanaga, S. Matsuoka, A. Ando, K.A. Tanaka and S. Nakai, Physical Review Letters 74, 3608 (1995).

24. C. Cherfils, S.G. Glendinning, D. Galmiche, B.A. Remington, A.L. Richard, S. Haan, R. Wallace, N. Dague and D.H. Kalantar, Physical Review Letters 83, 5507 (1999). 
a)

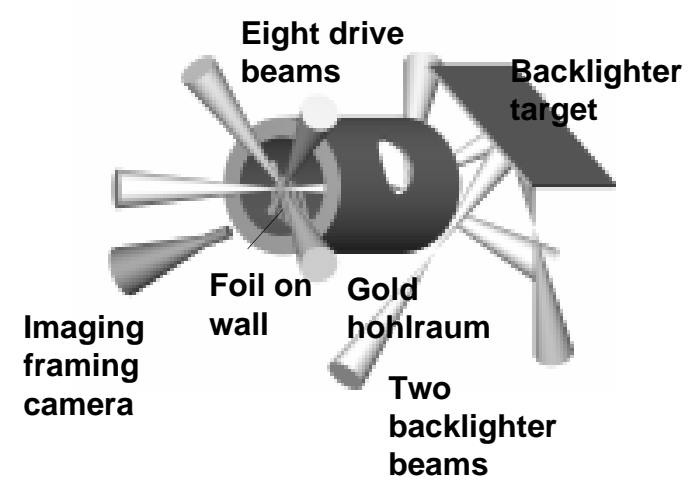

b)

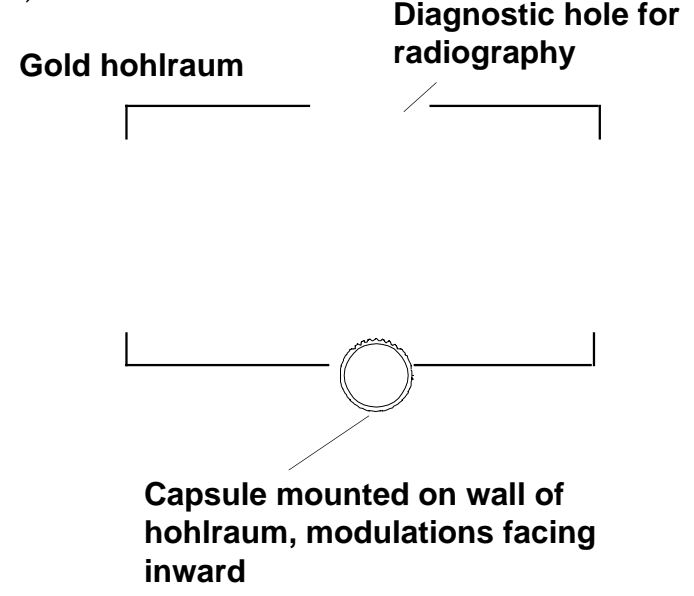

Figure 1 

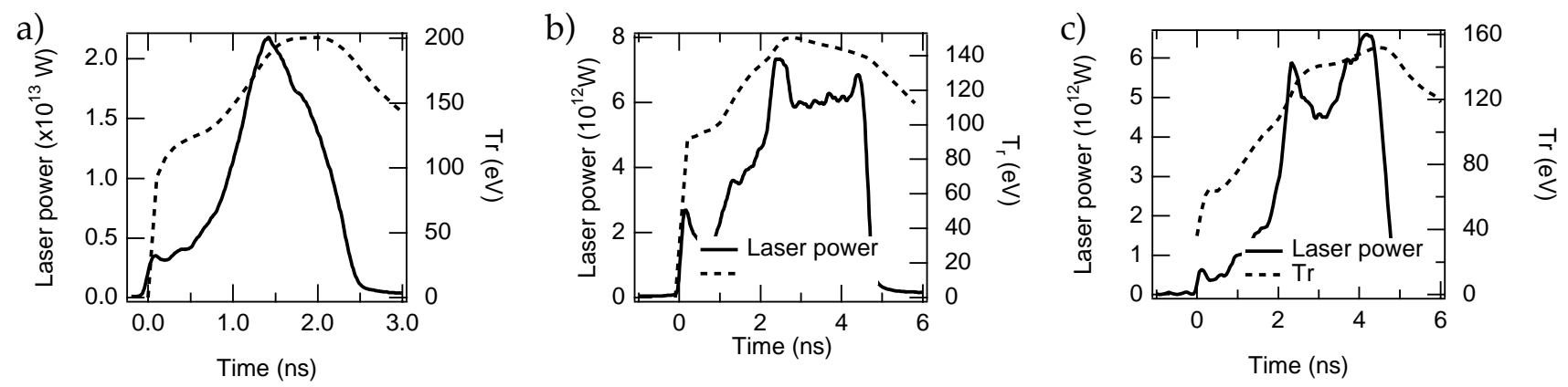

Figure 2 


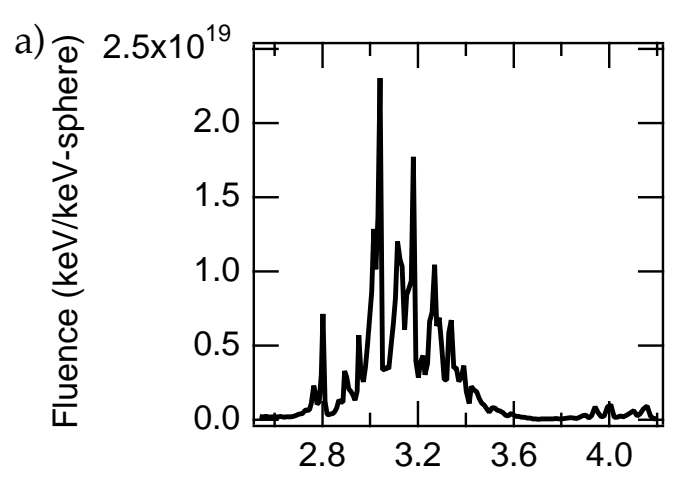

Photon energy (keV)

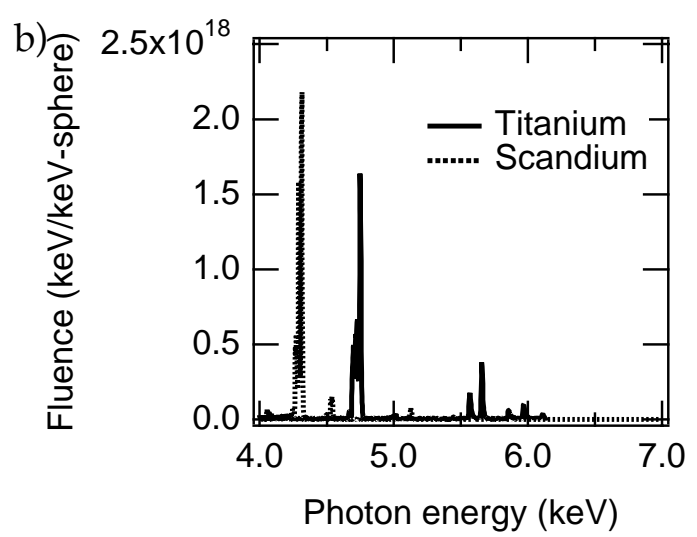

Figure 3 


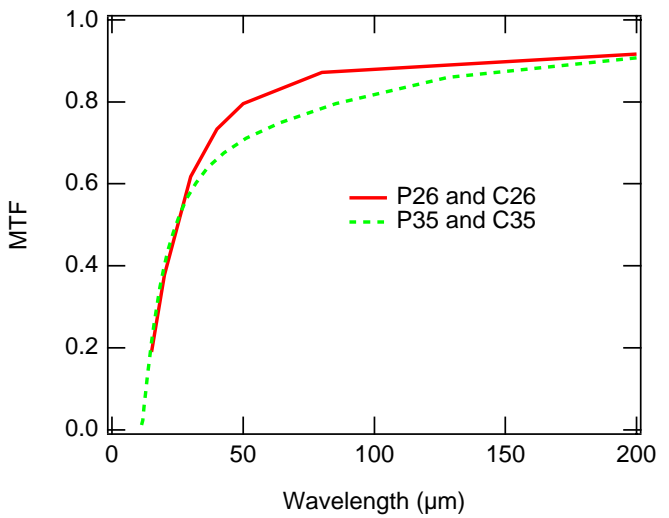

Figure 4 

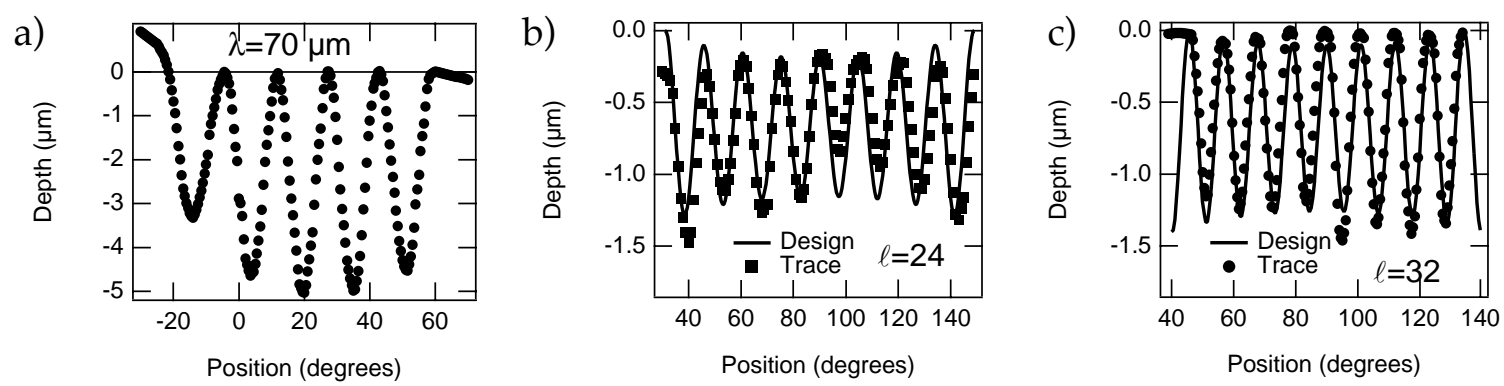

Figure 5 
a)

P26, 1.5 ns

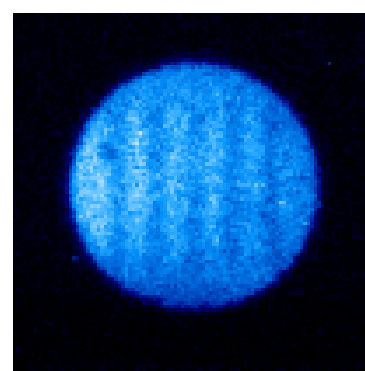

$\longmapsto 500 \mu \mathrm{m}$

c)

P35, 4.7 ns

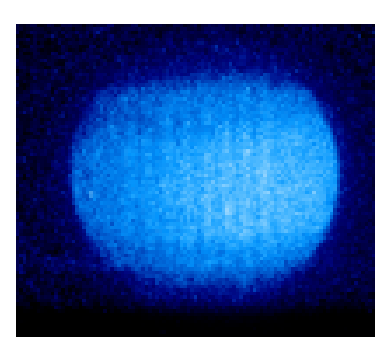

P26, 2.2 ns
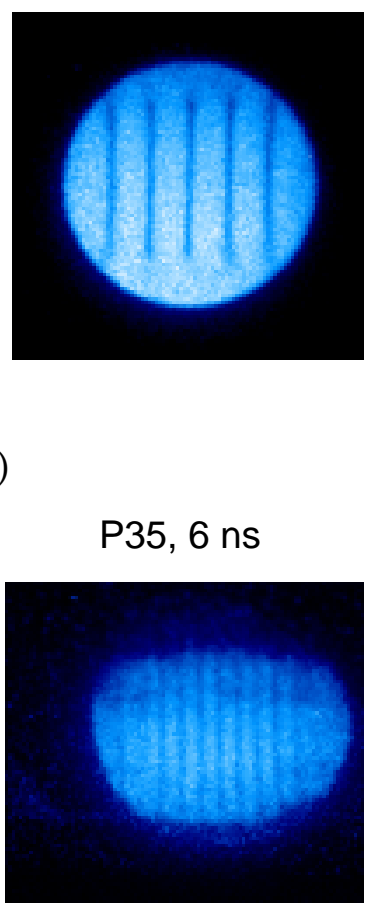

b)

C26, 1.5 ns
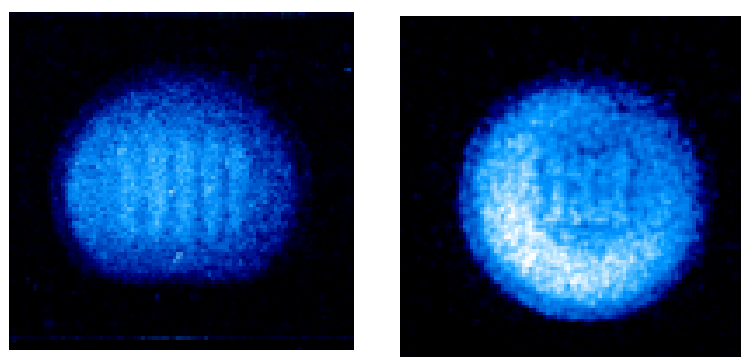

d)

C35, 4.5 ns

C35, $5.0 \mathrm{~ns}$
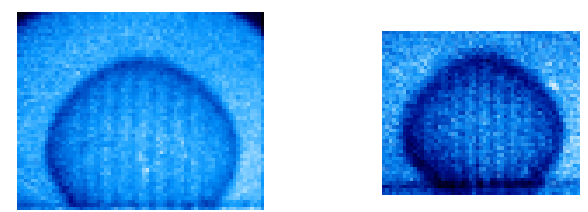

$500 \mu \mathrm{m}$

Figure 6 


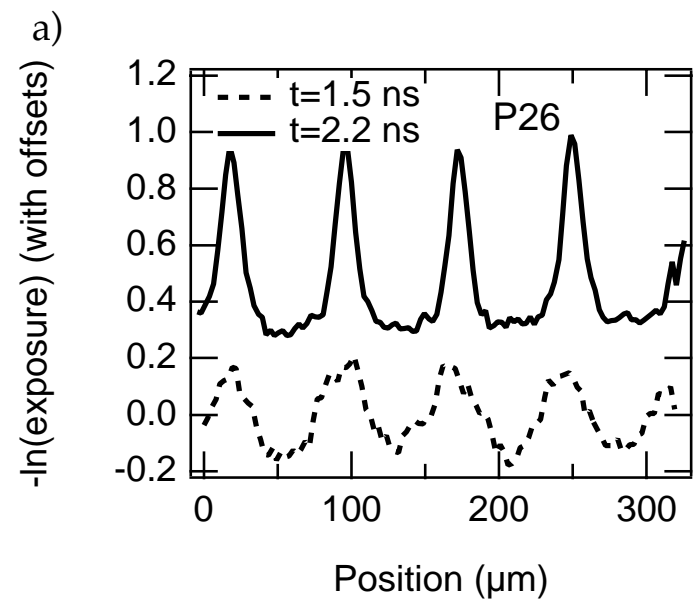

b)
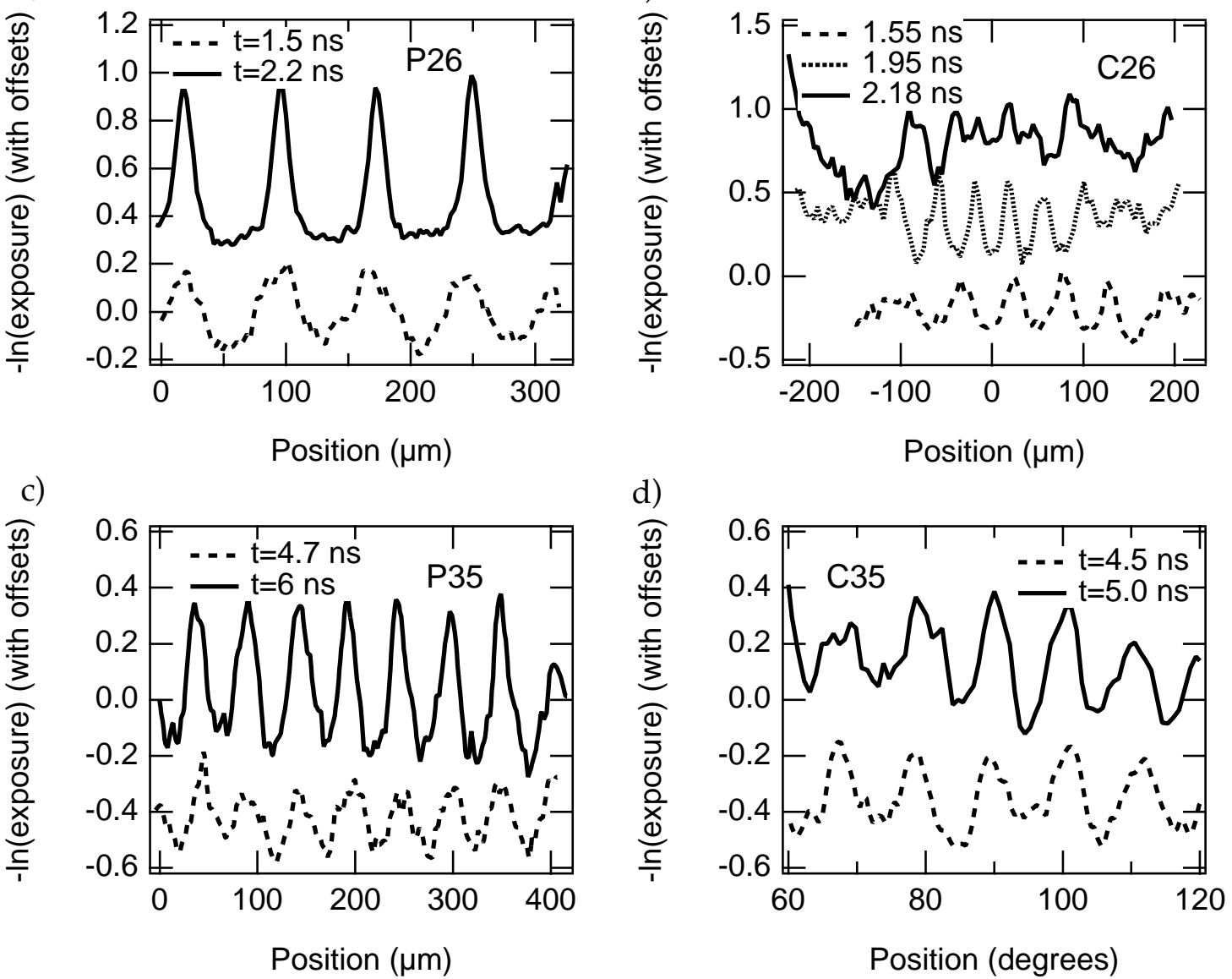

Figure 7 
a)

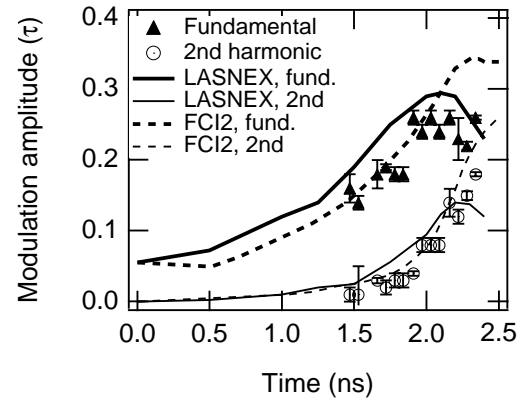

b)

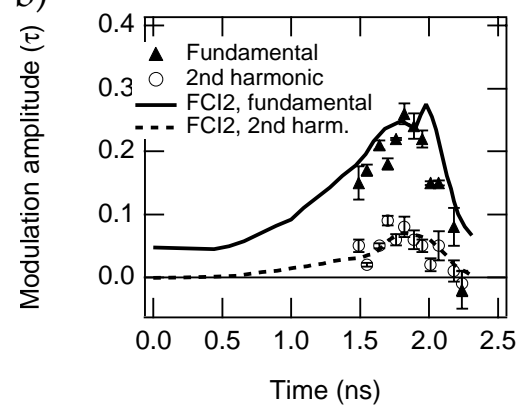

c)

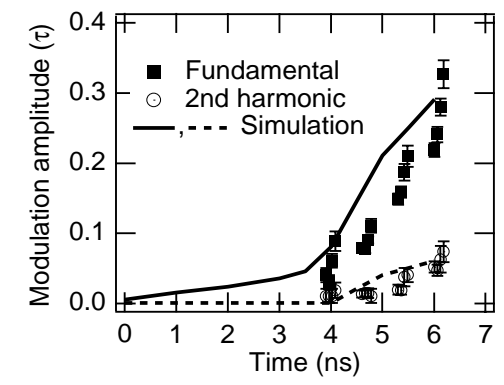

d)

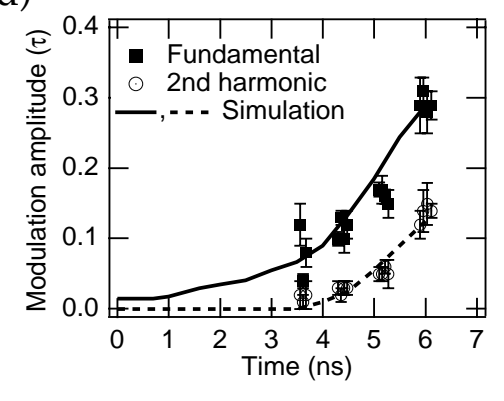

e)

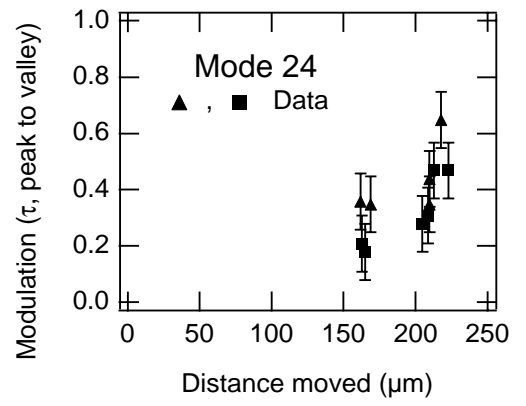

f)

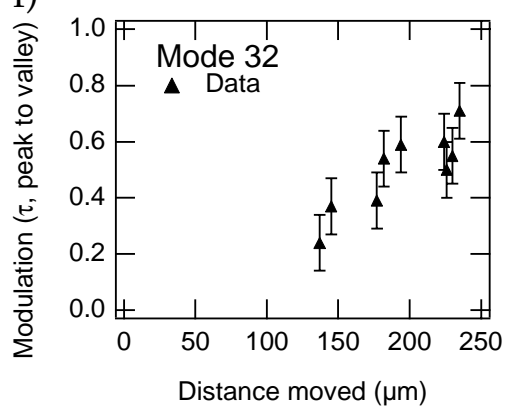

Figure 8 


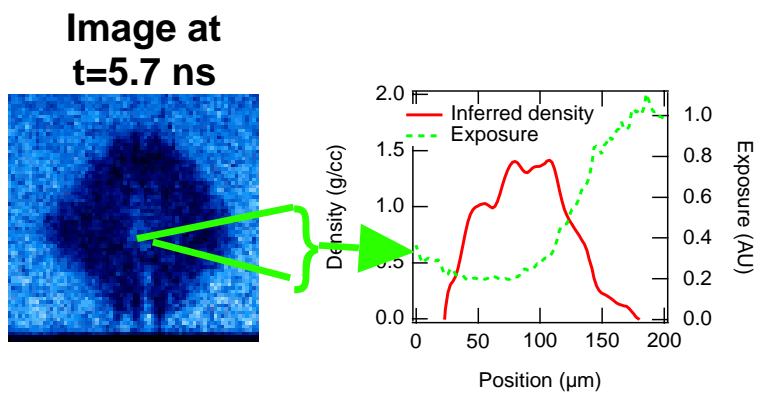

Figure 9 

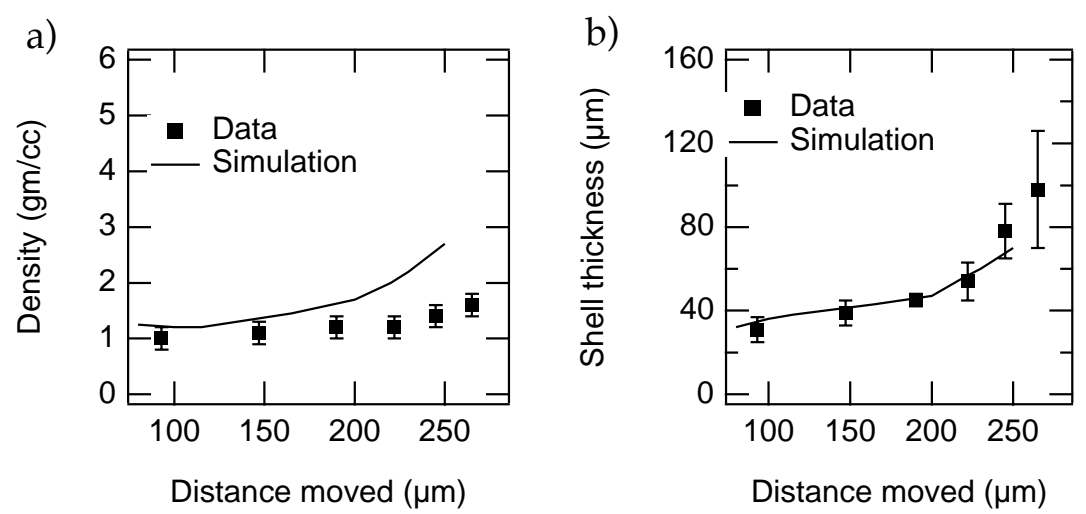

Figure 10 

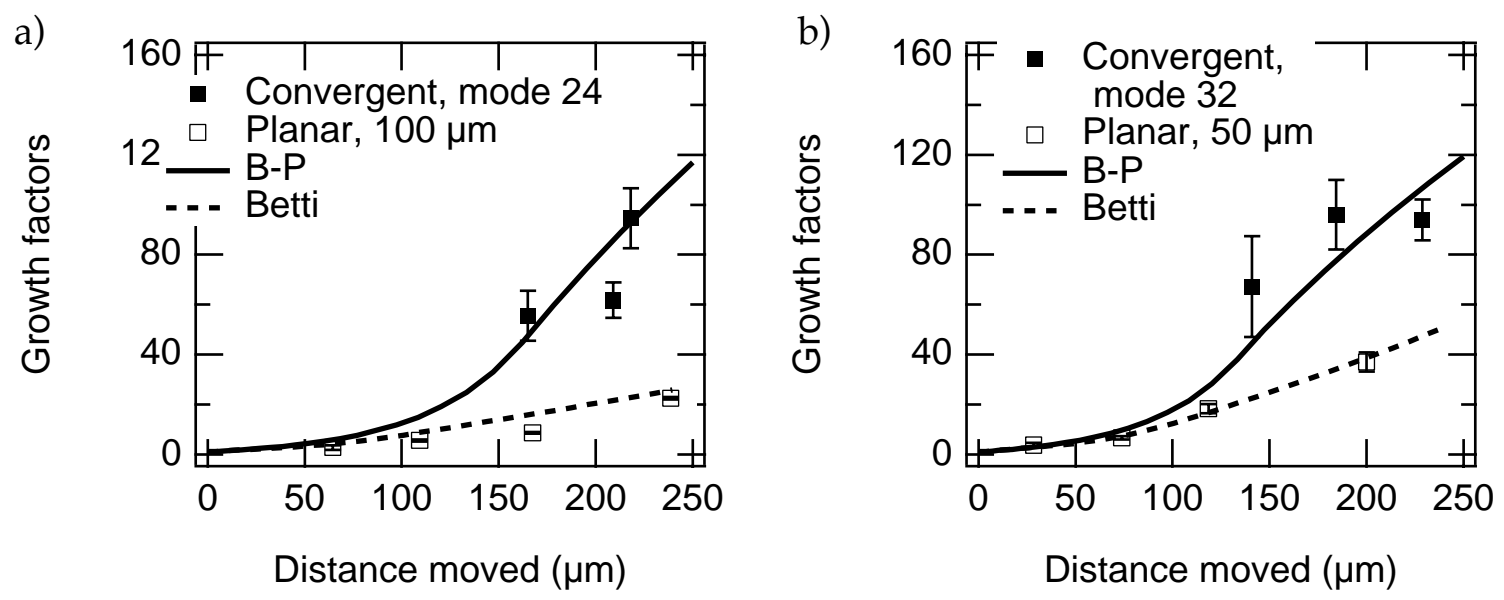

Figure 11 